\title{
A Reflex Electron Beam Discharge as a Plasma Source for Electron Beam Generation
}

\author{
CHRIS S. MURRAY, MEMBER, IEEE, JORGE J. ROCCA, MEMBER, IEEE, AND B. SZAPIRO
}

\begin{abstract}
A reflex electron beam glow discharge has been used as a plasma source for the generation of broad-area electron beams. An electron current of $120 \mathrm{~A}\left(12 \mathrm{~A} / \mathrm{cm}^{2}\right)$ was extracted from the plasma in $10 \mu \mathrm{s}$ pulses and accelerated to energies greater than $1 \mathrm{keV}$ in the gap between two grids. The scaling of the scheme for the generation of multikiloamp high-energy electron beams is discussed.
\end{abstract}

$\mathrm{W}$ E HAVE demonstrated that the plasma created by a reflex electron beam glow discharge constitutes an efficient source of thermal electrons for subsequent acceleration into intense broad-area electron beams. Electron currents of up to $120 \mathrm{~A}\left(12 \mathrm{~A} / \mathrm{cm}^{2}\right)$ were extracted from the plasma of the reflex discharge and accelerated in the gap between two grids at $\mathrm{keV}$ energies.

Several types of electron sources, including thermionic cathodes [1], photoelectric emitters [2], vacuum plasmas [3]-[5], and hollow cathode discharges [6]-[8], have been used and are currently being studied for broad-area electron beam generation. Low-pressure, high-voltage glow discharges have been demonstrated to generate high current density ( $>10 \mathrm{~A} / \mathrm{cm}^{2}$ ), broad-area electron beams [9]-[13]. In these glow discharges almost the entire discharge voltage drops in the cathode sheath [13], where the emitted electrons are accelerated to form the electron beam. Electron emission occurs following the bombardment by energetic ions and fast neutrals. Cold cathode glow discharge electron guns do not suffer the phenomenon of diode closure and are of simple construction. They have been shown to produce electron beam current densities $>10 \mathrm{~A} / \mathrm{cm}^{2}$ at energies of $50-90 \mathrm{keV} \mathrm{[10].} \mathrm{How-}$ ever, at energies $>100 \mathrm{keV}$ the electron beam current density is limited by frequent arcs to values on the order of $1 \mathrm{~A} / \mathrm{cm}^{2}[10]$.

Here we present a novel electron beam generation scheme in which two glow discharge electron guns are used in a reflex configuration to create a dense and cold plasma in a large volume. The thermal electrons created mainly by electron beam ionization are subsequently accelerated by an externally applied electric field in the gap between two grids to produce a broad-area electron beam. The electron beam current density and energy are inde-

Manuscript received September 16, 1987; revised May 31. 1988. This work was supported by the U.S. Air Force. B. Szapiro was supported by a fellowship from the Universidad Nacional de Buenos Aires.

The authors are with the Department of Electrical Engineering, Colorado State University, Fort Collins, CO 80523.

IEEE Log Number 8822661 pendently controlled by the voltage applied to the glow discharge guns and by the electric field sustained between the grids, respectively.

Fig. 1(a) is a schematic representation of the electron gun configuration used in the experimental demonstration of the concept reported here. Fig. 1(b) shows the electron gun in the cross-shaped stainless steel vacuum chamber. Aluminum cathodes $3 \mathrm{~cm}$ in diameter surrounded by ceramic tubes constitute two glow discharge electron guns that are placed $15 \mathrm{~cm}$ apart in a reflex configuration. Both electron guns are maintained at the same potential. At an operating pressure of 0.2 torr of helium the multikilovolt beam electrons produced by the glow discharge guns have a reaching distance that is several times longer than the distance between the guns. Consequently, the electrons can travel back and forth between the cathodes, generating a dense negative glow plasma. This is as follows: the electrons emitted by one of the cathodes are accelerated in the corresponding cathode sheath region and travel towards the opposite cathode, where they are reflected by the potential barrier presented by the cathode sheath. The beam electrons maintain this oscillatory motion until they become thermalized after suffering a large number of ionizing and exciting collisions or are scattered outside the plasma. In their oscillatory motion the beam electrons ionize the gas, creating an almost electric field-free plasma in which the secondary electrons created by ionization thermalize to a very low electron temperature $(<1 \mathrm{eV})$ [10], [14].

The process is similar to the operation of hollow cathode discharges. Hollow cathode discharges have been used as electron sources for broad-area beams [6]-[8]. However, in the reflex electron beam discharge the electron production efficiency can be several times larger. This is a consequence of the much larger secondary electron emission yield of materials when bombarded by $\mathrm{keV}$ ions, as compared to ions with a few hundred $\mathrm{eV}$ in hollow cathode discharges [18]. While in hollow cathode discharges the majority of the discharge current consists of ion flux bombarding the cathode, in high-voltage glow discharges with high electron yield cathode materials, it is not unusual for the electron beam current to be within a few percent of the total discharge current [10]. The enhanced production of energetic primary electrons results in a more efficient creation of thermal electrons. Also, the reflex glow discharge might have fewer constraints to be scaled to large areas because at multikiloelectronvolt 


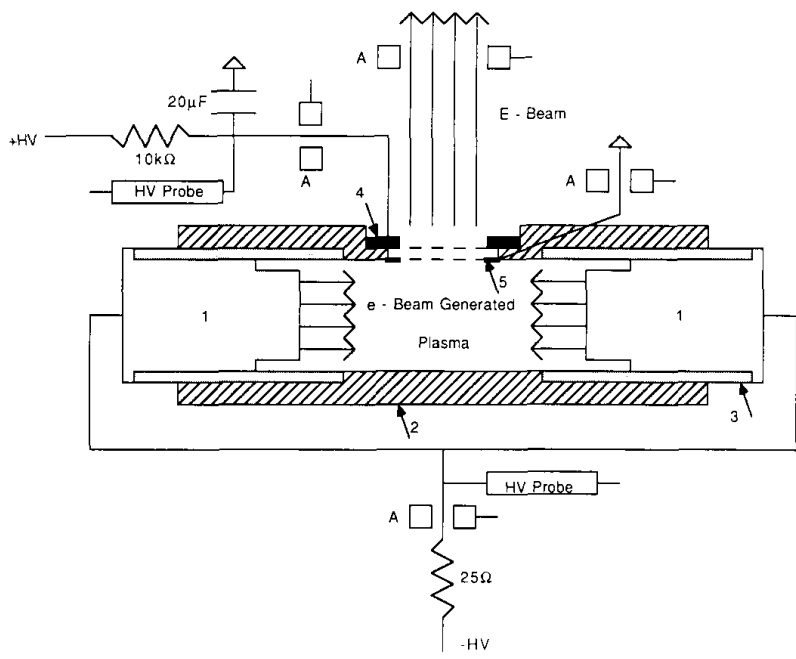

(a)

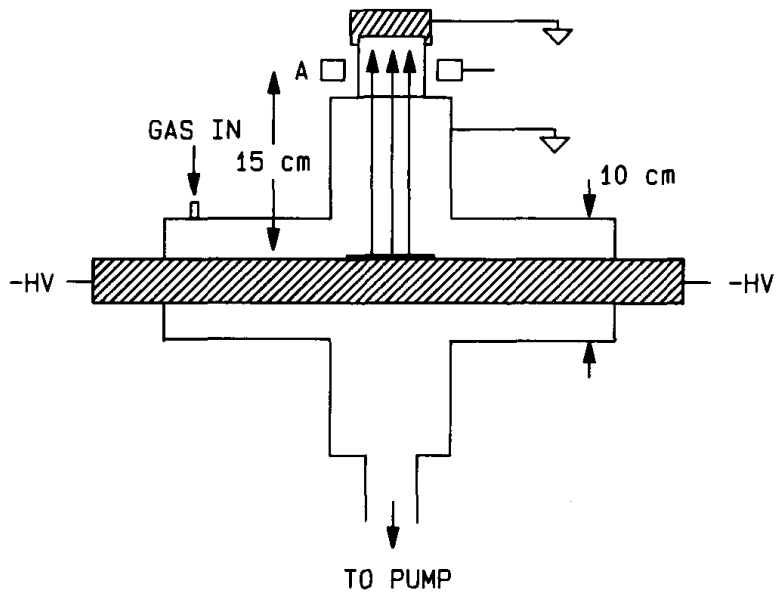

(b)

Fig. 1. (a) Schematic diagram of the electron gun and excitation circuit 1) Cylindrical glow discharge aluminum cathodes; 2 ) delrin support body; 3) ceramic tube; 4) and 5) stainless steel grids and metal supports. Pulse current transformers are identified with an $\mathbf{A}$. The vacuum enclosure is not shown. (b) Schematic diagram of electron gun in the cross-shaped stainless steel vacuum chamber. The current transformer used to measure the electron beam current is also shown.

energies and low helium pressures $(<0.5$ torr $)$ the reaching distance of the beam electrons is several meters and it is possible to create extended plasmas.

The electron guns of the reflex discharge were connected to a $50 \mathrm{nF}$ capacitor through a $25 \Omega$ ballast resistor and a triggered spark gap. The capacitor was negatively charged to voltages up to $9 \mathrm{kV}$. Thermal electrons from the discharge diffuse to the $0.25 \mathrm{~cm}$ gap between two grids, where they are accelerated to form a beam. The grids are rectangular with dimensions of $2 \mathrm{~cm}$ by $5 \mathrm{~cm}$ and are made of number 40 stainless steel mesh with a transmissivity of 40 percent. In most of our tests the inner grid was grounded and the external grid was directly connected to a $20 \mu \mathrm{F}$ capacitor as shown in Fig. 1(a). This capacitor was positively charged to voltages corresponding to the desired energy of the extracted electron beam up to $1.5 \mathrm{keV}$. The total current of the glow discharge guns, the inner grid, and the outer acceleration grid was monitored using three commercially available pulse current transformers. The variation of the voltage applied to the glow discharge guns and the acceleration voltage applied between the two grids was monitored using two commercially available high-voltage dividers. The entire electron gun structure was enclosed in a $10 \mathrm{~cm}$ diameter stainless steel chamber with several diagnostic ports and initially evacuated to a pressure of $10^{-3}$ torr by a rotary pump. Helium was continuously flown through the chamber, and the pressure was maintained at 0.2 torr by adjusting a needle valve. A small amount of oxygen ( 5 mtorr) was added to maintain the surface of the aluminum cathodes oxidized with the purpose of allowing a high emissivity in prolonged operation at high discharge current densities [15].

Above $1.5 \mathrm{kV}$ of acceleration voltage, an arc discharge was observed to develop between the positively biased acceleration grid and the metal walls of the vacuum envelope that surrounds the electron gun. By grounding the outer grid and negatively biasing the inner grid and the glow discharge electron guns with respect to the outer grid, we avoided this problem and were able to successfully operate the electron gun at acceleration voltages up to $5 \mathrm{kV}$. The reflex electron beam discharge was operated at discharge currents up to $100 \mathrm{~A}$ with pulse widths of 10 $\mu$ s. Fig. 2(a) shows the variation of the voltage applied to the guns and the corresponding total current of the reflex discharge. The discharge voltage at the time of the peak current, $70 \mathrm{~A}$, is $4 \mathrm{kV}$. If no voltage is applied to the external grid, practically all the current is collected by the inner grid, as shown by Fig. 2(b). When an acceleration voltage is applied to the outer grid, the current to the inner grid reverses sign and becomes an ion current, as shown in Fig. 2(c) for an acceleration voltage of $1200 \mathrm{~V}$. The electron current accelerated through the grid gap then equals the sum of the glow discharge cathode current and the inner grid current. The accelerated electron current was measured to be up to 1.5 times the glow discharge cathode current, as can be seen in Fig. 2(d). We observed a similar behavior in hollow cathode grid controlled electron guns [6]. As described by the theory of Zharinov et al. [16], it corresponds to grid-controlled plasma cathodes in which the wire spacing in the grids is larger than the width of the space charge layer near the anode.

Fig. 2(d) illustrates that the accelerated electron current pulse closely follows the discharge current pulse that provides the thermal electrons that diffuse into the acceleration gap between the grids to form the beam. The length of the electron beam current pulse is limited by the charge available in the capacitor that sustains the reflex discharge. Contrary to plasma guns in which an expanding plasma short-circuits the acceleration gap, the gun described here is in principle capable of dc operation. We have recently demonstrated long electron beam pulse generation $(70 \mu \mathrm{s})$ and dc operation of a grid-controlled hollow cathode plasma gun based on similar principles [6]. 


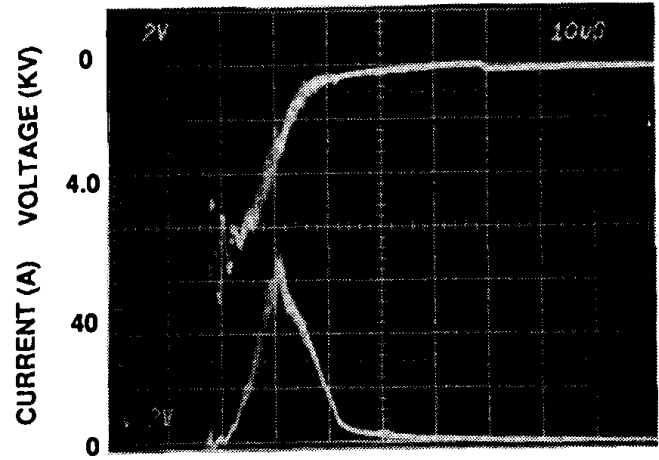

(a)

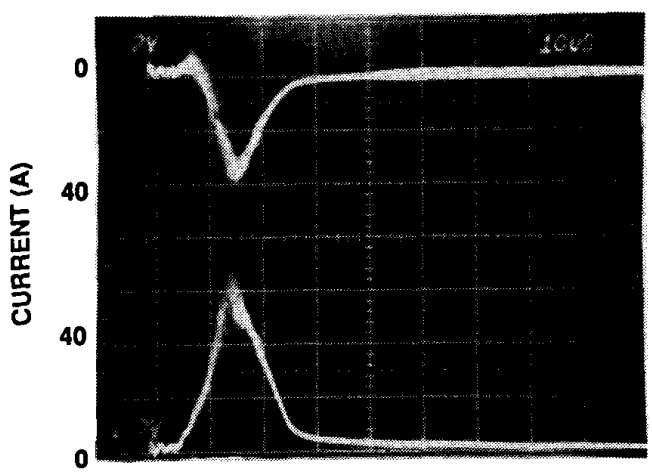

(c)

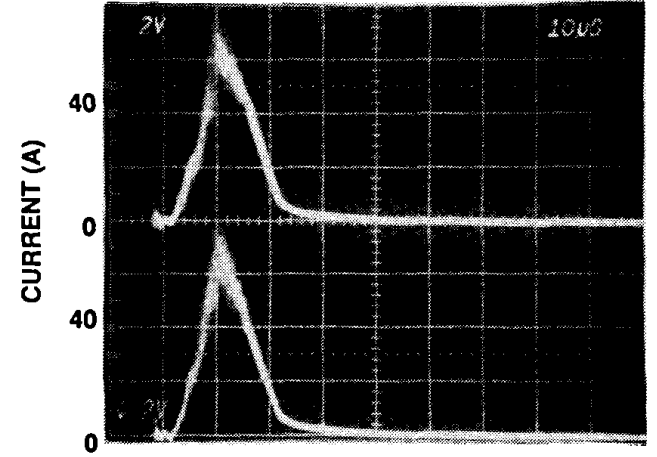

(b)

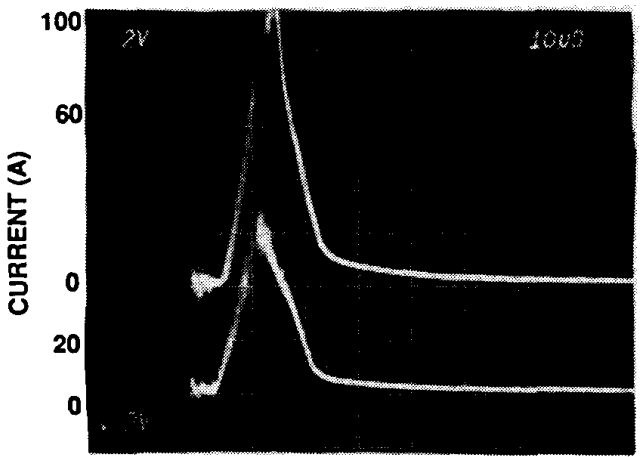

(d)

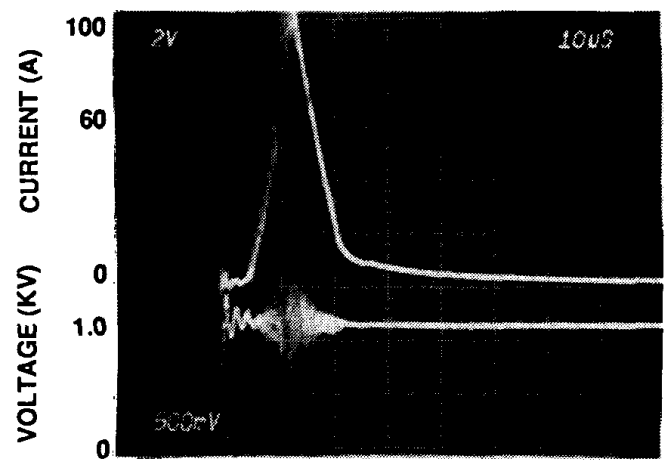

TIME (10us/div)

(e)

Fig. 2. (a) Upper trace: discharge voltage; lower trace: current of the reflex discharge. (b) Upper trace: inner grid current; lower trace: corresponding reflex discharge current pulse. Acceleration voltage $\mathrm{V}_{\text {accl }}=$ OkV. (c) Upper trace: inner grid current; lower trace: reflex discharge current $\mathrm{V}_{\mathrm{accel}}=1.2 \mathrm{kV}$. (d) Upper trace: accelerated (outer grid) electron current at $1.2 \mathrm{kV}$; lower trace: reflex discharge current. (e) Upper trace: accelerated electron current; lower trace: voltage across the acceleration gap. The helium pressure was 0.2 torr with 5 mtorr oxygen added.

Fig. 2(e) shows a $100 \mathrm{~A}\left(10 \mathrm{~A} / \mathrm{cm}^{2}\right)$ electron flux collected by the outer grid after having been accelerated to $1.2 \mathrm{keV}$ by the field maintained between the grids. Electron currents up to $120 \mathrm{~A}\left(12 \mathrm{~A} / \mathrm{cm}^{2}\right)$ were emitted by the $3 \mathrm{~cm}$ diameter reflex discharge and accelerated to form a well-collimated beam. The electron current density was measured using a pulse current transformer at $15 \mathrm{~cm}$ from the outer extraction grid, as shown in Fig. 1(b). At an accelerating voltage of $4 \mathrm{kV}$, approximately 40 percent of the electron current accelerated in the grid gap was measured by this current transformer. This percentage is in agreement with the transmissivity of the acceleration grid.

In this experiment the dimensions of the glow discharge and the values of the extraction voltage limited the discharge currents to $100 \mathrm{~A}$ and the electron beam current to $120 \mathrm{~A}$. Also, the maximum acceleration voltage applied 
was $5 \mathrm{kV}$. It should be possible to scale the concept discussed here to multikiloamp currents and high voltages $(100 \mathrm{kV})$. To create the reflex discharge reported in this work we employed glow discharge electron guns that in individual operation gave a maximum electron beam current of only $20 \mathrm{~A}$ [17]. To scale the concept to greater electron beam currents, the $3 \mathrm{~cm}$ in diameter guns could be replaced with larger diameter cathodes that have, with $50 \mathrm{kV}$ excitation, produced currents of up to $900 \mathrm{~A}[10]$. Two of these guns in a reflex configuration should be able to produce multikiloamp currents for subsequent acceleration to high energies in the gap between the two grids. As in other grid-controlled plasma guns that operate in a gaseous atmosphere, the maximum electron beam energy will ultimately be limited by Paschen or vacuum breakdown in the acceleration gap [8].

In summary, we have demonstrated the use of a reflex electron beam glow discharge as a plasma source for a grid-controlled electron gun. An electron current density of $12 \mathrm{~A} / \mathrm{cm}^{2}$ was extracted from the plasma and accelerated to energies greater than $1 \mathrm{keV}$ in $10 \mu \mathrm{s}$ pulses. The electron gun does not present diode closure, and it might be possible to scale it to produce multikiloamp electron beam currents at high energies.

\section{REFERENCES}

[1] K. Amboss, "Electron and ion beam science and technology," in Proc. 6th Int. Conf. Electrochem. Soc. (San Francisco). Princeton, NJ: Electrochem. Soc., 1974, pp. 497-517.
[2] C. H. Lee et al., IEEE Trans. Nucl. Sci., vol. NS-32, p. 3045, 1985; and C. H. Lee, Appl. Phys. Lett., vol. 44, p. 565, 1984.

[3] H. Shields, R. L. Sandstrom and J. I. Levatter, Appl. Phys. Lett., vol. 47, p. $681,1985$.

[4] S. Humphries, Jr., N. Savage, and D. M. Woodall, Appl. Phys. Lett. vol. 47, p. $468,1985$.

[5] R. J. Adler, G. F. Kiuttu, B. E. Simpkins, D. J. Sullivan, and D. E. Voss, Rev. Sci. Instr., vol. 52, p. 766, 1985.

[6] J. J. Rocca, B. Szapiro, and T. Verhey, Appl. Phys. Lett., vol. 50, p. 1334,1987

[7] A. V. Zharinov, Yu. A. Kavalenko, I. S. Roganov, and P. M. Tyuryukanov, Sov. Phys.-Tech. Phys., vol. 31, p. 39, 1986.

[8] J. R. Bayless and R. C. Knechtli, IEEE J. Quantum Electron., vol. QE-10, p. 213, 1974.

[9] G. W. McClure, Phys. Rev, vol. 125, p. 969, 1961.

[10] H. Ranea-Sandoval, N. Reesor, B. Szapiro, C. Murray, and J. J. Rocca, IEEE Trans. Plasma Sci., vol. PS-15, p. 361, 1987.

[11] B. O'Brien, Jr., Appl. Phys. Lett., vol. 22, p. 503, 1973.

[12] G. G. Isaacs, D. L. Jordan, and P. J. Dooley, J. Phys. E. Sci. Instrum., vol. 72, p. 115, 1979.

[13] S. A. Lee, L. U. Andersen, J. J. Rocca, M. Marconi, and N. Reesor, Appl. Phys. Lett., vol. 51, p. 409, 1987.

[14] Z. Yu, J. J. Rocca, G. J. Collins, and C. Y. She, Phys. Lett., vol. 96A, p. 125, 1983.

[15] J. J. Rocca, J. D. Meyer, M. Farrell, and G. J. Collins, J. Appl. Phys., vol. 52, p. 790, 1984

[16] A. V. Zharinov, Yu. A. Kovalenko, I. S. Roganov, and P. M. Tyuryukanov, Sov. Phys. -Tech. Phys., vol. 31, p. 413, 1986.

[17] B. Wernsman, H. F. Ranea-Sandoval, J. J. Rocca, and H. Mancini, IEEE Trans. Plasma Sci., vol. PS-14, p. 518, 1986.

[18] G. Carter and J. S. Colligon, Ion Bombardment of Solids. New York: Elsevier, 1968. 